\section{Schallemissionen aus Insektenohren: Hinweis auf aktives Hören?}

\author{
Manfred Kössl, Doreen Möckel, Melanie Weber und Ernst-August Seyfarth
}

\begin{abstract}
Zusammenfassung
Hörsinnesorgane von Insekten können otoakustische Emissionen produzieren. Derartige Schallemissionen aus dem Ohr gelten bei Wirbeltieren als Nebenprodukt einer aktiven und nichtlinearen cochleären Schallverstärkung durch spezialisierte Sinneszellen, die leise Schallschwingungen verstärkt und damit die Hörempfindlichkeit beträchtlich erhöht. Obwohl Wirbeltiere und Insekten andersartige Sinneszellen in ihrem Gehör aufweisen (Haarsinneszellen versus primäre Sinnesneurone mit einem Cilium), scheinen evolutiv konvergente Konstruktionsprinzipien der Mikromechanik des Ohrs vorzuliegen, um hohe Empfindlichkeit zu erzielen. Tympanalorgane von Insekten emittieren bestimmte otoakustische Emissionen, die auf nichtlinearen Eigenschaften des Sinnesorgans beruhen und - ähnlich wie die von Wirbeltieren - vom intakten Zellstoffwechsel abhängen. Allerdings ist für Tympanalorgane noch nicht geklärt, ob tatsächlich eine schnelle aktive Verstärkung durch die Sinneszellen vorliegt oder ob passive zelluläre Eigenschaften die nichtlineare Ohrcharakteristik ausmachen. Bei den antennalen Hörorganen von Fliegen hingegen wurde eine aktive Motilität des letzten Antennengliedes nachgewiesen. Wir zeigen am Beispiel der tympanalen Hörorgane von Heuschrecken und Nachtfaltern, dass die Emissionen frequenzspezifisch emittiert werden und durch elektrische Manipulation der Sinneszellen modifizierbar sind. Selbst das einfache Ohr bestimmter Nachtfalter, das nur eine einzige Sinneszelle aufweist, produziert deutliche Emissionen. Zurzeit ist weder bei Wirbeltieren noch bei Insekten zufriedenstellend geklärt, ob die für ein empfindliches Gehör essenzielle Nichtlinearität bzw. Verstärkung primär durch somatische Motilität der Sinneszellkörper bedingt wird oder durch aktive Bewegungsfähigkeit der Haarbündel bzw. Cilien der Sinneszellen. Weitere Untersuchungen am einfachen Modellsystem Insekt können hier helfen, offene Fragen zu klären.
\end{abstract}

\begin{abstract}
Do otoacoustic emissions from tympanal organs of insects indicate active hearing?

The tympanal hearing organs of insects produce otoacoustic emissions that can be recorded with the aid of a sensitive microphone. In vertebrates (including humans), such emissions are considered a by-product of active sound amplification through specialized sensory cells in the inner ear (i.e., the outer hair cells). Force-generation by these cells primarily amplifies faint sound and thus improves auditory sensitivity. As in vertebrates, the emissions from insect ears are based on non-linear mechanical properties of the sense organ. To achieve maximum sensitivity, convergent evolutionary principles were realized in the micromechanics of the ears - although vertebrates and insects possess different types of sensory cells in their auditory organs. Just as in vertebrates, otoacoustic emissions from insects ears depend on an intact metabolism, but so far in tympanal organs, it is not clear if amplification is achieved by active motility of the sensory neurons or if passive cellular characteristics cause the non-linear behavior. In the antennal ears of flies, however, active motility of the flagellum has been demonstrated. Here we review experiments on tympanal organs of grasshoppers and moths; we show that their otoacoustic emissions are produced in a frequency-specific way and can be modified by electrical stimulation of the sensory cells. Even the simple ears of notodontid moths produce distinct emissions, although they have just one auditory neuron. At present it is still uncertain, both for vertebrates and insects, if the non-linear amplification so essential for sensitive sound processing is primarily due to electromotility of the cell body of the sensory cells or to active movement of their hairbundles or cilium. Further experiments with the relatively simple ears of insects may help to answer these questions.
\end{abstract}

Key words: hearing organs; tympanal organ; otoacoustic emissions; non-linear amplification; electromotility
Otoakustische Emissionen Das Innenohr erzeugt Schallenergie

Sinnesorgane wandeln in ihren sensorischen Zellen spezifische physikalische Reizenergie in eine Änderung des Membranpotenzials um. Diese Umwandlung, auch als Reiztransduktion bezeichnet, basiert entweder auf intrazellulärer Reizaufnahme wie im Falle visueller Reize oder, in mechanisch sensitiven Sinnessystemen, auf direkter Aktivierung von Membranproteinen, die meist an einen Ionenkanal gekoppelt sind. Derartige Transduktionsmechanismen arbeiten normalerweise nur in eine Richtung.

Aus diesem Grund war die Überraschung groß, als David Kemp im menschlichen äußeren Gehörgang Schallereignisse messen konnte, die offensichtlich vom Innenohr erzeugt wurden (Kemp 1978). Er stimulierte das Ohr mit kurzen akustischen Klickreizen und zeigte, dass Schallwellen einer spezifischen Tonfrequenz mit einer bestimmten Verzögerungszeit im Gehörgang auftraten. Ursprünglich wurden diese Ereignisse auch als „Kemp-Echos“ bezeichnet. Allerdings haben sie ihren Ursprung nicht in einer echoartigen Reflektion am Trommelfell oder im Mittelohr, sondern hängen von einem physiologisch intakten Innenohr ab. Eine Reihe nachfolgender Studien wies nach, dass die äußeren Haarzellen des Innenohrs von Säugetieren essenziell für diese Schallgenerierung sind (z.B. Kakigi et al. 1998). Während die inneren Haarzellen massiv von Dendriten der Hörnervneurone innerviert sind und ihre Hauptfunktion in einer Weiterleitung der Hörinformation an auditorische Zentren im Gehirn zu sehen ist, besitzen die äußeren Haarzellen nur wenige Hörnervsynapsen, sind jedoch direkt von efferenten Axonen innerviert, die eine zentrifugale Informationsübertragung vom Hirnstamm an das Innenohr ermöglichen. In der Zellmembran äußerer Haarzellen wurde ein Protein, das Prestin, entdeckt, das spannungsabhängige Konformationsänderungen durchführt und bei Depolarisation bzw. Hyperpolarisation des Membranpotenzials eine Verkürzung bzw. Verlängerung des Zellkörpers bewirkt (Übersicht: Dallos und Fakler 2002). Damit ist die äußere Haarzelle in der Lage, bei Schallreizung Kraft zu erzeugen, welche die Auslenkungsamplitude von Basilarmembran und Corti-Organ im Innenohr deutlich erhöhen kann. Die spannungsabhängige Motilität der äußeren Haarzellen wird als Grundlage des sogenannten ,cochleären Verstärkers“ angesehen (Übersicht: Geleoc und Holt 2003), der bei Säugetieren für eine Verbesserung der Innenohrempfindlichkeit um etwa 40-60 dB sorgt (Faktor 100-1000). 


\section{Exkurs 1}

\section{Charakterisierung von otoakustischen Emissionen}

Man unterscheidet zwei Arten von otoakustischen Emissionen (OAE). Sogenannte ,place dependent“ OAE enthalten spezifische Tonfrequenzen. Dementsprechend sind die Emissionen einem ganz bestimmten Frequenzabbildungsort im Innenohr zuzuordnen. $\mathrm{Zu}$ den placedependent OAE gehören sowohl Emissionen, die bei externer Schallreizung des Innenohrs auftreten (wie Klick-evozierte OAE oder Dauerton-evozierte OAE), als auch spontane OAE (SOAE), die auch ohne externe Tonreizung messbar sind. Die SOAE stellen den direktesten Hinweis dar, dass im Innenohr aktiv Schall generiert werden kann. SOAE sind bei vielen Versuchspersonen in Form eines sehr engen Amplitudenmaximums bei einer bestimmten Tonfrequenz messbar (s. die Abbildung, links unten). Oftmals treten auch mehrere SOAE pro Innenohr auf. SOAE sind meist sehr leise, mit Amplituden unterhalb unserer Hörschwellensensitivität, sodass sie nicht hörbar, wohl aber mit einem empfindlichen Mikrofon messbar sind. In manchen Fällen erreichen sie aber einen Schalldruck von bis zu 20 dB SPL und werden dann auch hörbar. Sie treten in unserem Bereich besten Hörens, zwischen 100-11000 Hz auf. Als Ursache für SOAE wird spontane Motilität von Haarzellen angesehen. Um allerdings die Frequenzspezifität dieser OAE erklären zu können, sind zusätzliche Mechanismen nötig, z.B. die Entstehung stehender Wellen an Diskontinuitäten der Cochleastruktur (siehe Abbildung).

Auch für die Erzeugung evozierter OAE spezifischer Frequenz sind derartige Zusatzvoraussetzungen nötig. Evozierte OAE können sich in SOAE umwandeln und umgekehrt. Dies macht deutlich, dass derselbe Mechanismus verantwortlich ist. Modelliert man OAE-Erzeugung in Form eines Rückkopplungssystems, dann unterscheiden sich beide Arten von OAE lediglich im Verstärkungsfaktor in der Rückkopplungsschleife.

Bei Amphibien, Reptilien und Vögeln gibt es auch deutlich ausgeprägte SOAE (siehe dazu die Abbildung; Frosch: Long et al. 1996; Reptilien: Manley 2006; Schleiereule: Taschenberger und Manley 1997). Hauptunterschied zum Säuger ist deren Begrenzung auf Frequenzen unterhalb von ca. $10 \mathrm{kHz}$. SOAE dieser Frequenz findet man bei der Schleiereule. Außerdem sind hier SOAE in ihrem Amplitudenspektrum weniger scharf ausgeprägt als bei Säugern. Bei Säugern sind SOAE bis etwa $62 \mathrm{kHz}$ dokumentiert (Fledermaus: Kössl 1994), was zeigt, dass im Säugerinnenohr eine extrem schnelle Schallverstärkung stattfinden kann, passend zum Hörvermögen vieler Säugetiere, das in den Ultraschallbereich ausgeweitet ist.
Eigenschaften frequenzanalysierender Algorithmen, üblicherweise wird der FFT(Fast-Fourier-Transform) Algorithmus verwendet, tauchen in einem gemessenen Frequenzspektrum die Klirranteile im regelmäßigen Abstand zu den Stimulusfrequenzen auf. Verwendet man zwei Reintonstimuli unterschiedlicher Frequenz $(\mathrm{f} 1, \mathrm{f} 2)$, dann sind die Verzerrungsprodukte bei Frequenzen von $(\mathrm{n}+1) \mathrm{f} 1-\mathrm{nf} 2$ und $(\mathrm{n}+1)$ f2-nf1 messbar. In den bislang untersuchten

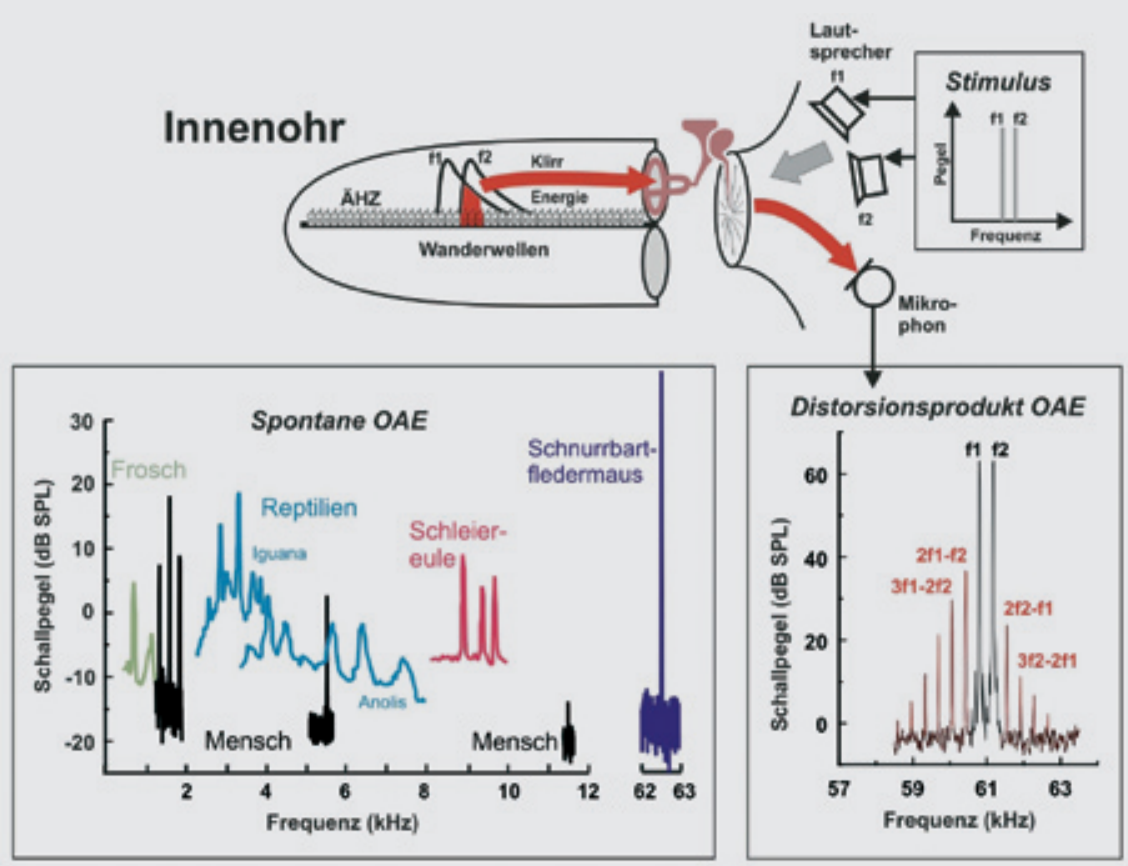

Abb. Exkurs 1: OAE bei Wirbeltieren. Spontane OAE (Abbildung unten links) sind ohne Schallreizung mit einem Mikrofon am Trommelfell messbar und treten in Form von Amplitudenmaxima bei spezifischen Tonfrequenzen auf. Distorsionsprodukt OAE (unten rechts) entstehen bei Reizung mit zwei Reintönen in Form zusätzlicher Maxima (in rot) an definierten Frequenzen im gemessenen Amplitudenspektrum. Sie werden in der Überlappungszone der beiden Reinton-Wanderwellen auf der Basilarmembran durch nichtlineare Verstärkung in den äußeren Haarzellen (ÄHZ) erzeugt (siehe Schema oben).

Eine andere Art otoakustischer Emissionen sind sogenannte ,wave-dependent“ OAE. Dies sind evozierte Emissionen, die bei Reizung mit mehreren Reintönen von unterschiedlicher Frequenz entstehen. Sie bestehen aus Klirranteilen, auch „Distorsionsprodukt-OAE“ (DPOAE) genannt, die aufgrund nichtlinearer Eigenschaften der Schallverarbeitung im Innenohr erzeugt werden. Der cochleäre Verstärker arbeitet nichtlinear - leise Schallreize werden höher verstärkt als laute. Dadurch kann sich eine Verzerrung der Signalform ergeben, die in einem Frequenzspektrum in Form von zusätzlichen Frequenzanteilen erfassbar ist (s. Abbildung, rechts unten). Aufgrund der
Gehörorganen ist die 2f1-f2 OAE am lautesten (siehe Abbildung, rechts unten). Klirren ist der Preis, der für eine hochempfindliche Schallverstärkung zu zahlen ist. Während viele technische Systeme, wie HiFi-Anlagen, Klirren dann erzeugen, wenn sie übersteuert werden und in Sättigung geraten, produziert das Innenohr Klirren vor allem für leise Eingangsschallpegel, bei denen der cochleäre Verstärker maximal aktiv ist. Im Gegensatz zu ,placedependent OAE", die - wenn sie überhaupt auftreten - meist nur auf eine Frequenz beschränkt sind, sind DPOAE, bei Wahl geeigneter Stimulusfrequenzen, im gesamten Hörbereich eines Tieres messbar. $\gg>$ 


\section{Fortsetzung Exkurs 1}

Sie bieten deshalb sehr viel umfassendere Information über cochleäre Verstärkungsprozesse. Aus diesem Grund werden sie auch zunehmend im klinischen Bereich, z.B. bei der Untersuchung von Hörschäden bei Neugeborenen, eingesetzt. Es ist wichtig zu betonen, dass jedes nichtlineare System Frequenzverzerrungen erzeugen kann, unabhängig davon, ob die Nichtlinearität durch aktive zelluläre Prozesse oder durch passive Systemeigenschaften bedingt ist, wie sie z.B. in einer übermäBigen Auslenkung des Trommelfells oder der Mitttelohrknöchelchen bei extrem lautem Schall vorliegen könnten. Im Falle des Innenohrs von Wirbeltieren sind, wie eine Reihe von Studien zeigen, sensitive DPOAE jedoch eindeutig durch aktive und stoffwechselabhängige Verstärkungsprozesse in den äußeren Haarsinneszellen bedingt.

Otoakustische Emissionen sind ein Nebenprodukt dieses cochleären Verstärkers und ermöglichen es, seine Funktion detailliert zu untersuchen (siehe Exkurs 1). Da otoakustische Emissionen nicht nur bei Säugetieren sondern auch bei anderen Vertebraten auftreten, deren auditorische Haarzellen kein elektromotorisch wirksames Prestin enthalten, sind in diesen Fällen andere zelluläre Verstärkungsprozesse involviert (siehe unten).

\section{Die Gehörorgane von Insekten}

Viele Insekten haben ein gutes Hörvermögen, das zur Wahrnehmung von Beute oder Feinden und zur innerartlichen Kommunikation eingesetzt wird. Im Vergleich zu empfindlichen Wirbeltierohren sind die oft winzigen Insektenhörorgane etwa um 20-30 dB (Faktor 10-15) weniger empfindlich. Anders als bei den Nichtsäugern unter den Wirbeltieren ist ihr Hörvermögen allerdings nicht auf relativ tiefe Tonfrequenzen unterhalb von $10 \mathrm{kHz}$ beschränkt, sondern reicht bei vielen Insektenarten bis weit in den Ultraschallbereich. Das gilt für viele Nachtfalter und beispielsweise auch für bestimmte südamerikanische Laubheuschrecken, die mit Gesängen von $130 \mathrm{kHz}$ den Hochfrequenzrekord für Schallaussendung bei Arthropoden halten (Montealegre-Z et al. 2006). Zu ihrem Gehör ist zwar noch nichts bekannt, aber es ist zu vermuten, dass sie ihre eigenen Gesänge auch hören.

Die Struktur von Hörorganen der Insekten unterscheidet sich stark vom Wirbeltierinnenohr. Man unterscheidet Tympanalorgane, bei denen die Sinnesneurone direkt an oder in der Nähe eines Trommelfells sitzen, von Gehörantennen, an deren Basis die Sinnesneurone ringförmig angeordnet sind. Tympanalorgane nehmen Schalldruck bis in den Ultraschallbereich war und sind prinzipiell in der Lage, auch Schall in Form von otoakustischen Emissionen zu erzeugen, da sie über ein steif aufgespanntes Trommelfell verfügen. Sie finden sich am Körper oder in den Beinen von Heuschrecken und Nacht- faltern. Antennenorgane hingegen nehmen im akustischen Nahfeld Luftmolekülbewegungen wahr und befinden sich am Kopf von Fliegen und Mücken.

Bei Wanderheuschrecken (Abbildung 1A) sind etwa 80 Sinnesneurone zu einem peripheren Sinnesganglion zusammengefasst (sog. „Müllersches Organ“). Wie bei allen Insektenhörorganen sind die Sinnesneurone skolopidiale Mechanorezeptoren mit einem sensorischen Cilium, das über einen massiven Wurzelapparat im Dendriten des Neurons verankert ist (Abbildung 1B). Der Bereich der Cilienspitze, der in eine Kappe eingepasst ist, die von einer Kappenzelle abgesondert wird, ist für die Schalltransduktion verantwortlich. Im Ganglion des Müllerschen Organs liegen die Sinneszellsomata, deren Dendriten in mehreren Strängen verschiedene Ansatzpunkte auf dem Trommelfell erreichen. Sie sind dort über ihre Kappenzellen an das Tympanum gekoppelt. Das Trommelfell weist dickere und dünnere Bereiche auf, die bei akustischer Reizung mit tiefen versus hohen Tonfrequenzen jeweils maximal auslenkbar sind. Die Tonfrequenz, auf welche die Sinneszellen optimal reagieren, hängt also vom Ansatzpunkt ihrer Dendriten auf dem Trommelfell ab. So befindet sich zum Beispiel das pyriforme (,,birnenförmige") Vesikel als Ansatzstruktur für eine Gruppe von Sinneszellen auf einem Bereich dünner Trommelfellmembran (Abbildung $1 \mathrm{~A})$; und entsprechend reagieren diese Zellen bevorzugt auf hohe Tonfrequenzen ab etwa $10 \mathrm{kHz}$. Andere Dendriten enden auf dickeren Stellen des Tympanums und sind
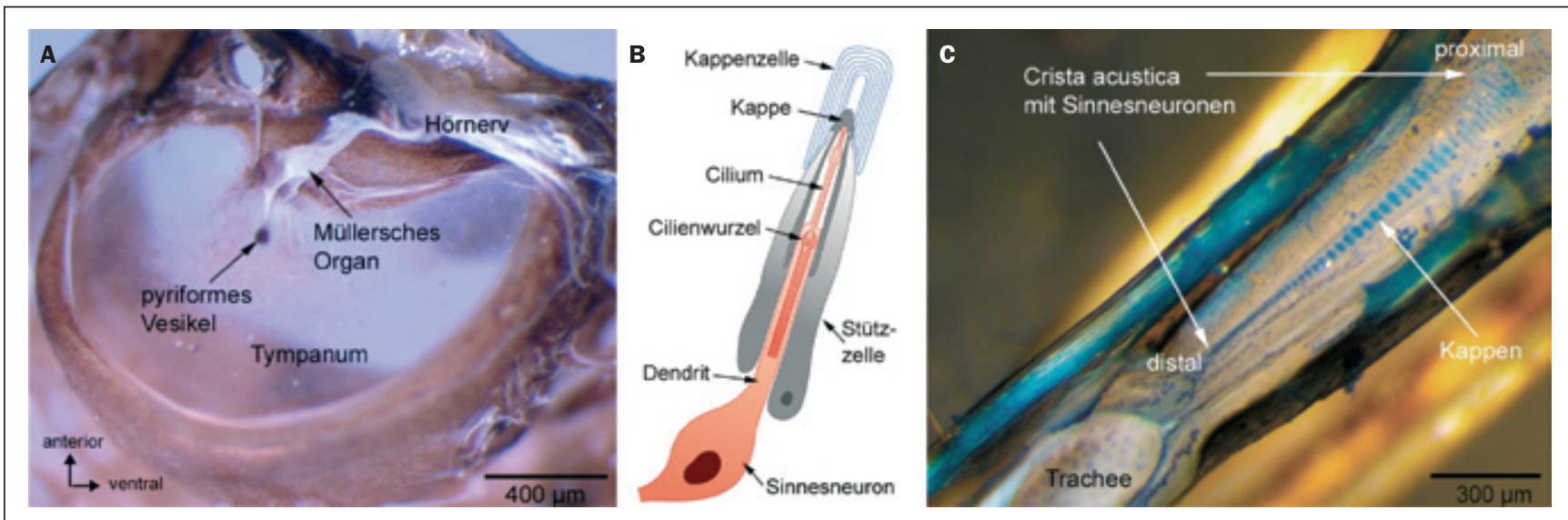

Abb. 1: Wichtige anatomische Kennzeichen der Tympanalorgane von Heuschrecken. (A) Ohr aus dem 1. Abdominalsegment einer Wanderheuschrecke (Locusta migratoria) von innen gesehen. Das relativ große Trommelfell (Tympanum) ist in einem Cuticularing aufgespannt. Am Rand sitzt das sensorische Ganglion (Müllersches Organ), von dem aus die Sinneszelldendriten zu unterschiedlich dicken Bezirken des Tympanums auslaufen. Die Dendriten im pyriformen Vesikel inserieren in einem relativ dünnen (hier hellen) Membranbezirk. (B) Schematische Darstellung eines Skolopidiums mit primärer Sinneszelle und Hilfszellen (Stütz- und Kappenzelle). Der Dendrit der Sinneszelle bildet in seinem distalen Teil ein Cilium aus. (C) Tympanalorgan einer tropischen Laubheuschrecke (Mecopoda elongata) nach Öffnung der Vorderbeintibia und Anfärbung mit Methylenblau. Die Crista acustica aus ca. 40 skolopidialen Sinneszellen liegt dorsal auf einer Hörtrachee und ist Teil eines größeren mechanosensorischen Organkomplexes im Insektenbein. 
für Tieffrequenzwahrnehmung zuständig. Im Tympanalorgan der Wanderheuschrecke findet also eine mechanische Frequenzfilterung und Erfassung von unterschiedlichen Tonfrequenzen statt, ähnlich wie dies auch bei Wirbeltieren der Fall ist (Michelsen 1971; Römer 1976; Windmill et al. 2005).

Eine tonotope Abbildung der Tonfrequenzen entlang einer sich kontinuierlich in ihren mechanischen Filtereigenschaften ändernden Struktur, wie sie zum Beispiel die Basilarmembran der Wirbeltiercochlea darstellt, findet sich im Tympanalorgan von Laubheuschrecken (Abbildung 1C). Hier sitzen die skolopidialen Sinneszellen in einem langgezogenen Sinnesorgan, der Crista acustica, im Inneren der Vorderbeintibia. Diese „Beinohren“" werden akustisch angeregt über eine Schalleintrittsöffnung im Thorax der Tiere. Eine Tracheenröhre (,,akustische Trachee") leitet den Schall dann in die Beine bis zum Sinnesorgan. Nahe beim Sinnesorgan befinden sich zwar Tympana, die aber nicht direkt von den Dendriten kontaktiert werden und vermutlich weniger der Schallanregung als vielmehr dem Druckausgleich dienen. Die skolopidialen Sinnesneurone mit ihren dendritischen Kappen sind senkrecht zur Längsrichtung der Crista acustica angeordnet, und in ihren Abstimmeigenschaften ist eine tonotope Frequenzverarbeitung nachweisbar (Oldfield 1988; Stumpner 1996). Neurone, die am besten auf hohe Frequenzen reagieren, liegen distal im Organ, solche, die auf tiefe Frequenzen reagieren, befinden sich proximal. Ein Blick auf das Organ verrät auch gleich, welche morphologischen Strukturen für diese tonotope Anordnung verantwortlich sein könnten, denn von proximal nach distal werden die dendritischen Kappen zunehmend kleiner (Abbildung 1C) und können damit vermutlich besser auf hohe Frequenzen reagieren. Dies schließt natürlich nicht aus, dass hier noch andere, sich tonotop ändernde Sinneszellcharakteristika vorliegen.

\section{Klirrende Tympanalorgane}

Aufgrund einer nichtlinearen Schallverstärkung im Innenohr von Wirbeltieren (d.h. leise Schallsignale werden mehr verstärkt als laute) kommt es zu einer Verformung der Wellenform der Signale und zur Erzeugung von Klirranteilen, die das Ohr verlassen und am Trommelfell messbar sind (Distorsionsprodukt Otoakustische Emissionen: DPOAE; siehe Exkurs 1). Bei Schallstimulation mit Reintönen erzeugen auch Tympanalorgane deutliche DPOAE (Abbildung 2), die in vieler Hinsicht vergleichbare Eigenschaften zu denjenigen aus Wirbeltierinnenohren haben

\section{Exkurs 2}

\section{Messung von DPOAE aus dem Ohr von Laubheuschrecken}

Zur Messung von DPOAE bei MecoD/A-Wandlern im PC erzeugt und nach Lautstärkeregelung und Endverstärkung auf zwei Lautsprecher verschaltet. Die Trennung beider Kanäle ist wichtig zur Verhinderung von Verzerrungsprodukten im schallerzeugenden System. Das Schallsignal, welches sowohl die beiden Stimuli als auch DPOAE enthält, wird mit einem Mikrofon gemessen und nach Verstärkung in einen A/D-Wandler eingespeist. Schallerzeugender und schallerfassender Kanal sind in einem Koppler vereint, dessen Spitzenöffnung auf die Dimension der Eingangsöffnung der akustischen Trachee (Spirakel) von Mecopoda angepasst ist. poda werden zwei Reintonsignale von
Der Vorteil dieser Messanordnung besteht darin, dass der Koppler fest am prothorakalen Spirakel angebracht ist und das Tympanalorgan mit der Crista acustica, das sich in der Tibia der Vorderbeine befindet, frei zugänglich für experimentelle Manipulationen bleibt.

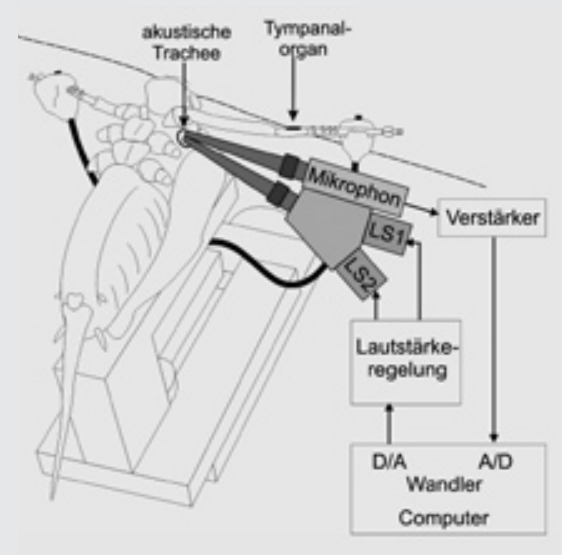

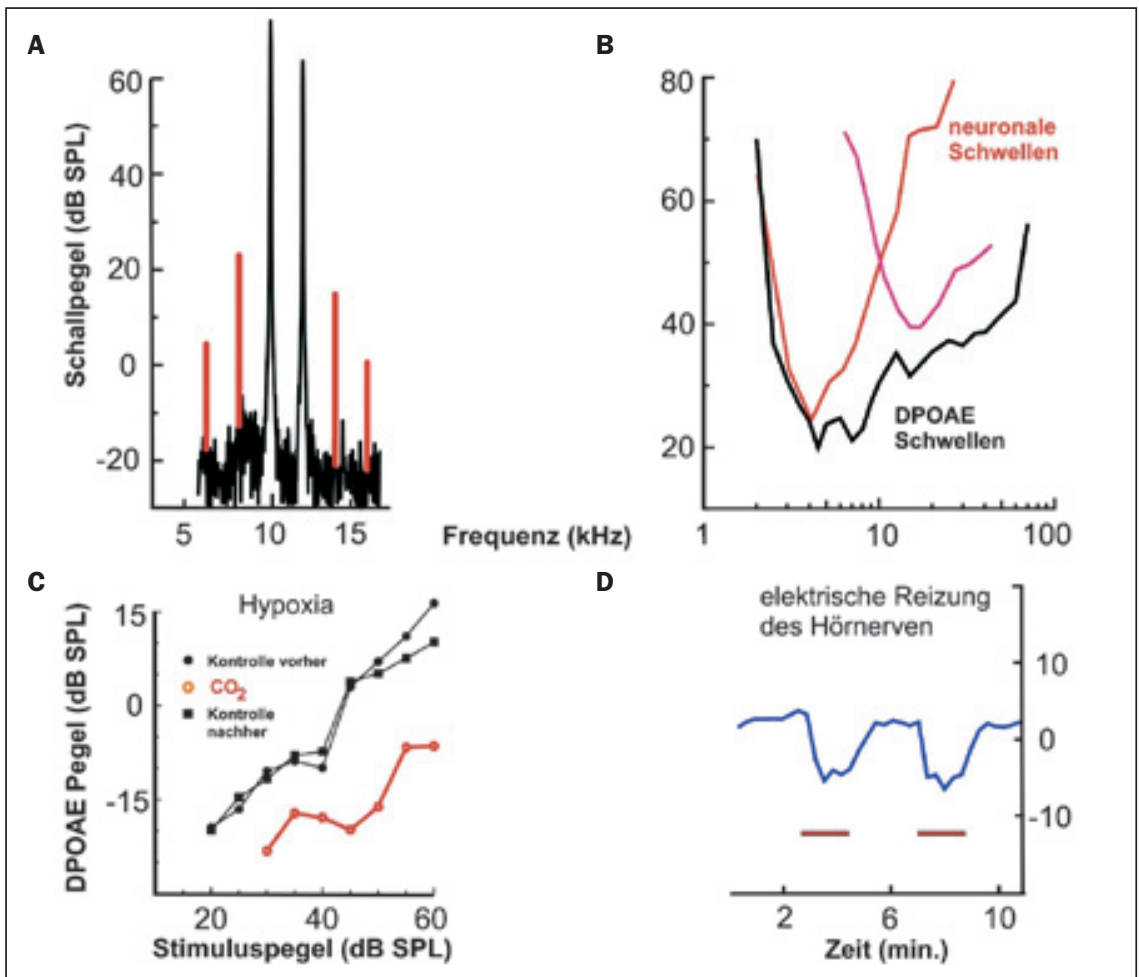

Abb. 2: DPOAE-Messungen am Tympanalorgan der Wanderheuschrecke. (A) DPOAE-Spektrum bei akustischer Reizung mit zwei Tönen von 10 und 12 kHz; die hier rot markierten Verzerrungsprodukte erreichen Schalldrucke, die sich deutlich vom Grundrauschen abheben. (B) Hörschwellenkurve, die für 2 Neuronengruppen bestimmt wurde (nach Römer 1976) und Schwellenschalldrucke zur Auslösung von DPOAE im Hörbereich der Tiere (nach Kössl und Boyan 1998). (C) Der Abfall der DPOAE-Pegel bei $\mathrm{CO}_{2}$-Behandlung (Hypoxia) demonstriert die Bedeutung von aktivem Stoffwechsel für die Ausbildung von otoakustischen Emissionen (aus Kössl und Boyan 1998). (D) Kurze elektrische Reizung des Hörnerven, in dem die Axone der primären Sinneszellen verlaufen, verursacht eine vorübergehende Reduktion des Emissionspegels (nach Möckel et al. 2007). 


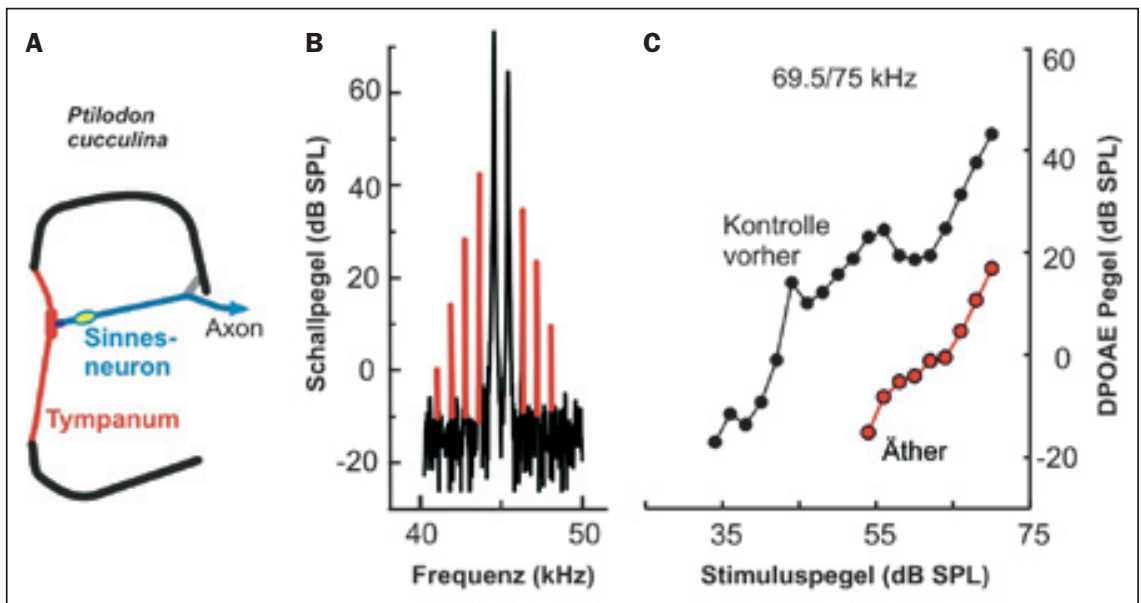

Abb. 3: Das metathorakale Tympanalorgan der Motte Ptilodon (Notodontidae) hat nur ein auditorisches Neuron; dennoch produziert das Ohr deutliche DPOAE im hochfrequenten Bereich. (A) Schematisierte Darstellung des Organs, in dem der Dendrit einer skolopidialen Sinneszelle an das Tympanum gekoppelt ist. (B) Spektrum der DPOAE (rot markiert) mit Tonreizen im Bereich von $45 \mathrm{kHz}$. (C) Wachstumsfunktionen vor und nach Betäubung mit Äther; die beiden Tonstimuli lagen bei $69.5 \mathrm{kHz}$ (f1) und $75 \mathrm{kHz}$ (f2) (verändert nach Kössl et al. 2007).

(Kössl und Boyan 1998; Coro und Kössl 1998). Messbar sind diese Emissionen mit Hilfe von Sonden, die auf den Durchmesser des Tympanums angepasst sind und einen Stimulationskanal mit zwei Lautsprechern sowie einen Aufnahmekanal mit einem Mikrophon enthalten (zur Methode siehe auch Exkurs 2). Bereits bei leisen Schallpegeln nahe der Hörschwelle von Heuschrecken oder Nachtfaltern lassen sich DPOAE induzieren, wobei die 2f1-f2-Emission am lautesten ist. Dies macht deutlich, dass die Bewegung des Tympanums auch an der Hörschwelle nichtlinear ist. Bestimmt man für unterschiedliche Stimulusfrequenzen den Stimuluspegel, der ausreicht, um eine DPOAE bestimmter Amplitude (z.B. -10 dB SPL) zu induzieren, kann man DPOAESchwellenkurven errechnen (Abbildung 2B). Sie erlauben Aussagen zur frequenzspezifischen Sensitivität der nichtlinearen Mechanik des Tympanalorgans und gleichen den neuronalen Schwellendaten. Man kann also, ähnlich wie bei Säugern und insbesondere beim Menschen, auch bei Insekten DPOAE-Messungen zur objektiven Hörschwellenbestimmung verwenden.

Insekten-DPOAE sind anfällig für Manipulationen, die den physiologischen Zustand des Tiers verändern, wie bestimmte Anästhetika oder eine Hypoxie (Abbildung 2C). Auch bei Säugetieren kommt es zu einer deutlichen Abnahme der DPOAE-Pegel bei derartigen Eingriffen. Die Effekte werden dort auf eine Blockierung des cochleären Verstärkers zurückgeführt. Bei Insekten ist noch unbekannt, ob die Sinneszellen und potenzielle Verstärkungsmechanismen von solchen Manipulationen betroffen sind. Die Tatsache, dass skolopidiale Sinnesneurone im Gegensatz zu Haarsinneszellen ein eigenes Axon haben, das direkt zum Zentralnervensystem zieht, bietet die Möglichkeit, in einiger Entfernung zum Sinnesorgan die Axone elektrisch zu stimulieren, ohne dass dessen Mechanik beeinträchtigt wird. So kann getestet werden, ob z.B. eine induzierte Veränderung des Membranpotenzials Auswirkungen auf die Erzeugung von otoakustischen Emissionen hat. In der Tat kommt es während elektrischer Stimulation zu reversiblen Änderungen der DPOAEAmplitude (Abbildung 2D). Dies ist ein gänge in den Sinnesneuronen selbst an der Erzeugung der Emissionen beteiligt sind.

Die Zahl der Sinneszellen in einem Tympanalorgan kann je nach Spezies stark variieren. Die einfachsten Organe finden sich bei bestimmten Nachtfaltern wie Ptilodon cucullina oder Phalera sp. aus der Gruppe der Notodontidae. Hier wird das Trommelfell über eine elastische Verbindung zwischen dem Zentrum der Membran und dahinterliegenden Skelettelementen gespannt, und nur eine einzige Sinneszelle sitzt an der Verbindung zwischen Spannelement und Tympanum (Abbildung 3A). Entsprechend dieser strategisch wichtigen Position sollte diese Zelle auf jegliche Auslenkungen des Tympanums empfindlich reagieren. Falls die skolopidiale Sinneszelle über aktive Verstärkerkomponenten verfügt, sollte sie auch in der Lage sein, die Mechanik des direkter Hinweis, dass mechanische Vor-
Tympanums direkt zu beeinflussen. Selbst das simple Ohr von Notodontiden sendet prominente DPOAE aus (Abbildung 3B), deren Wachstumscharakteristik vergleichbar ist mit den DPOAE von komplexeren Tympanalorganen und von Wirbeltierohren. Bei Betäubung des Tieres mit Äther sinken die DPOAE-Amplituden deutlich ab (Abbildung 3C), was für die Beteiligung aktiver metabolischer Vorgänge an der Emissionserzeugung spricht.

Genaue Informationen zum Ort der Erzeugung von DPOAE lassen sich am Tympanalorgan der Wanderheuschrecke mit einer lokalen Läsion der Anheftungspunkte von Sinneszelldendriten am Tympanum ermitteln (Abbildung 4). Die Auslenkung des Tympanums erfolgt frequenzspezifisch in Form von Wanderwellen (Abbildung 4A; Windmill et al. 2005). Am pyriformen Vesikel werden maximale Auslenkungen durch hohe Tonfrequenzen ab etwa $12 \mathrm{kHz}$ induziert (im hier gezeigten Beispiel 26 $\mathrm{kHz}$ ). An einer anderen Ansatzstelle von Sinneszelldendriten, dem ,folded body“, sind tympanale Auslenkungen nur mit tiefen Tonfrequenzen erzielbar (im hier angegebenen Fall 3,3 kHz; Abbildung 4B). Durchtrennt man per mechanischer Mikroläsion die Sinneszelldendriten zwischen dem pyriformen Vesikel und dem sensorischen Ganglion (Abbildung 4C), dann beobachtet man eine drastische Reduktion von hochfrequenten DPOAE. Bei zusätzlicher Läsion des „folded body“ und des dahinterliegenden sensorischen Ganglions sind die DPOAE über den gesamten Frequenzbereich deutlich reduziert (Abbildung 4D). Dies macht deutlich, dass die mechanosensorischen Neurone frequenzspezifisch an der DPOAE-Aussendung beteiligt sind.

\section{Vibrierende Antennenorgane}

Für Tympanalorgane ist damit aber noch nicht ganz geklärt, dass tatsächlich ein aktiver auditorischer Verstärkungsmechanismus in den Sinneszellen vorliegt und wie ein solcher Verstärker beschaffen ist. Die Arbeitsgruppe von Martin C. Göpfert (derzeit in Köln) wies mit laservibrometrischen Messungen am Antennenorgan von Drosophila nach, dass in diesem Organ tatsächlich mechanische Energie erzeugt wird. Ein überzeugender Beweis einer solchen aktiven Krafterzeugung sind spontane Vibrationen der Hörantennen bei Frequenzen unterhalb von etwa $1000 \mathrm{~Hz}$. Sie lassen sich nach pharmakologischer oder genetischer Manipulation induzieren (Göpfert uund Robert 2003; Übersicht: Göpfert 2007). Derartige Vibrationen der 
Antennen erzeugen zwar nicht ausreichend Schalldruck, um als spontane OAE messbar zu sein (was am Fehlen eines Trommelfells liegt); die zellulären Mechanismen könnten jedoch ähnlich zu denjenigen in Tympanalorganen sein. Bei Drosophila sind Proteine aus der TRP-Familie (Transient-ReceptorPotential) für die sensorische Transduktion verantwortlich (Übersicht: Kernan 2007), ähnlich wie bei Vertebraten. Durch Deletion der Gene für den Transduktionskanal oder daran gekoppelter Proteine kann die spontane Vibration deutlich erhöht oder reduziert werden (Göpfert und Robert 2003; Göpfert et al. 2005, 2006). Die skolopidialen Sinneszellen von Drosophila enthalten interessanterweise auch ein prestinhomologes Protein (Weber et al. 2003). Ob dieses Protein, ähnlich wie das Prestin in den äußeren Haarzellen der Säugercochlea, für eine mechanische Krafterzeugung relevant ist, ist nach gegenwärtiger Lage der Dinge eher unwahrscheinlich. In skolopidialen Sinnesorganen scheint es so zu sein, dass die Krafterzeugung über Proteine stattfindet, die mit dem Transduktionsapparat assoziiert sind.

\section{Vergleich aktiver Ohrmechanik in Wirbeltieren und Insekten}

Bei Reptilien und Vögeln beruhen aktive mechanische Verstärkungsprozesse im Innenohr nicht auf Zellkörpermotilität, sondern auf einer Motilität des Haarbündels (Stereovilli) sensorischer Haarzellen. Dementsprechend sind die Stereovilli auch verantwortlich für die Erzeugung spontaner OAE (Manley et al. 2001; Übersicht: Manley 2001). Bei Säugern hingegen scheint eine auf Prestin basierende Motilität des Zellkörpers der äußeren Haarzellen der entscheidende Faktor für eine cochleäre Verstärkung zu sein, was allerdings nicht ausschließt, dass auch Verstärkungsprozesse in den Stereovilli vorliegen können (Übersicht: Fettiplace und Hackney 2006). Prestin reagiert bei Veränderung des Membranpotenzials nach erfolgter Transduktion mit einer schnellen Konformationsänderung und dadurch verkürzt oder verlängert sich das Zellsoma. Dieser Prozess ist sehr schnell und kann auch höchsten Tonfrequenzen folgen. Beim Meerschweinchen wurde entsprechende Elektromotilität bis zu etwa $70 \mathrm{kHz}$ gemessen (Frank et al. 1999), und es ist zu vermuten, dass manche echoortenden Säuger, deren Hörbereich bis über $200 \mathrm{kHz}$ reicht, auch in diesem Grenzbereich einen cochleären Verstärker benutzen, der auf Prestin basiert. Wie im Exkurs 1 erwähnt, sind spontane OAE bei einer Fledermausspezies bis zu etwa 62 $\mathrm{kHz}$ messbar (Abbildung 5 und Exkurs 1). Verstärkungsprozesse in den Stereovilli hingegen, die Interaktionen zwischen dem Transduktionskanalmolekül und Aktin/ Myosin-ähnlichen Proteinen umfassen, wie sie auch wichtig für Adaptationsvorgänge sind, arbeiten langsamer. So sind aktive Haarbündelbewegungen, Adaptationsvorgänge oder auch elektrisch induzierte OAE bei den bislang untersuchten Nichtsäugern auf Frequenzen unterhalb ca. $3 \mathrm{kHz}$ beschränkt (Manley et al. 2001; Fettiplace et al. 2001). Übrigens haben potenzielle aktive Haarbündelprozesse bei Säugern ein ähnliches Frequenzlimit (Ricci et al. 2005; Übersicht: LeMasurier und Gillespie 2005). Entsprechend hat der Hörbereich von Nichtsäugern eine deutlich tiefere obere Grenzfrequenz (Abbildung 5). Spitzenreiter

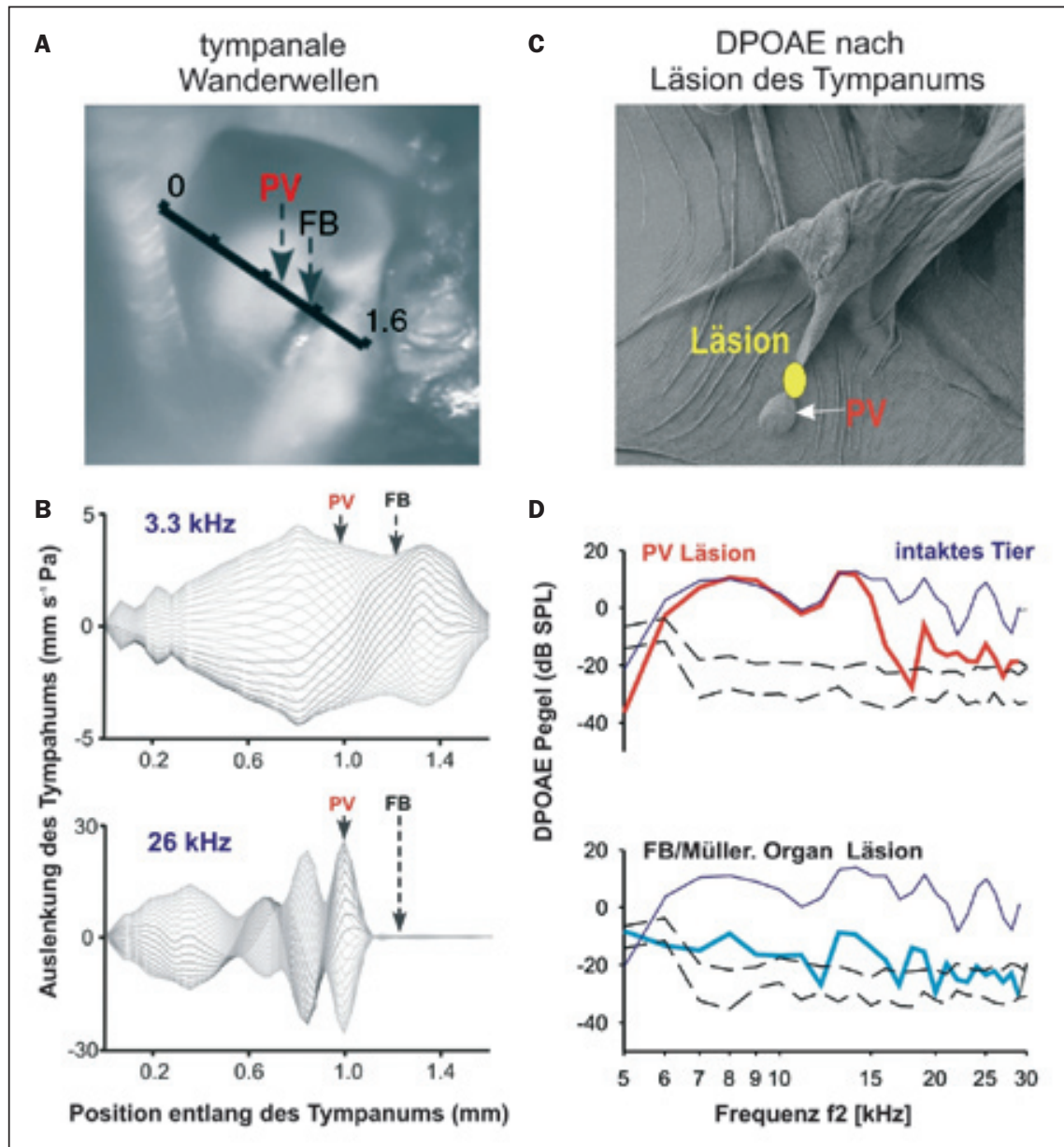

Abb. 4: Locusta migratoria; Wanderwellen und DPOAE vor/nach Läsionen. (A) Aufblick auf das Tympanum, die durchgezogene Linie gibt Messpositionen an (in $\mathrm{mm}$ ) für die Erfassung der Bewegung des Tympanums mit einem Laservibrometer. Markiert ist die Position des pyriformen Vesikels (PV) und des „folded body“ (FB). (B) Schallreizung mit Reintönen erzeugt ein wanderwellenähnliches Bewegungsmuster entlang der Messpositionen. Bei Stimulation mit tieffrequenten Signalen $(3.3 \mathrm{kHz})$ werden sowohl PV als auch FB deutlich ausgelenkt. Hochfrequente Signale (26 kHz) führen zu einem Amplitudenmaximum am PV, ohne dass der FB ausgelenkt wird. (C) Blick auf die Innenseite des Tympanums mit dem Müllerschen Organ und PV. Der PV wurde durch eine Läsion vom Müllerschen Organ entkoppelt. (D) Sogenanntes DP-gram: Dargestellt sind DPOAE-Pegel für Schallstimuli konstanter Lautstärke und unterschiedlicher Frequenz (f2). Das Frequenzverhältnis f2/ f1 war auf 1.08 eingestellt. Nach Läsion des PV sinken DPOAE oberhalb von etwa $15 \mathrm{kHz}$ fast auf den Pegel des Umgebungsrauschens ab (gestrichelte Linien: Mittelwert und SD des Rauschens). Bei einer nachfolgenden Läsion des FB und Müllerschen Organs am selben Präparat sinken die DPOAE-Pegel im gesamten Frequenzbereich. Dies macht deutlich, dass hochfrequente DPOAE nur bei intakter Verbindung der Sinneszellen zum PV entstehen. (A,B verändert nach Windmill et al. 2005; C,D verändert nach Möckel et al. 2007). 


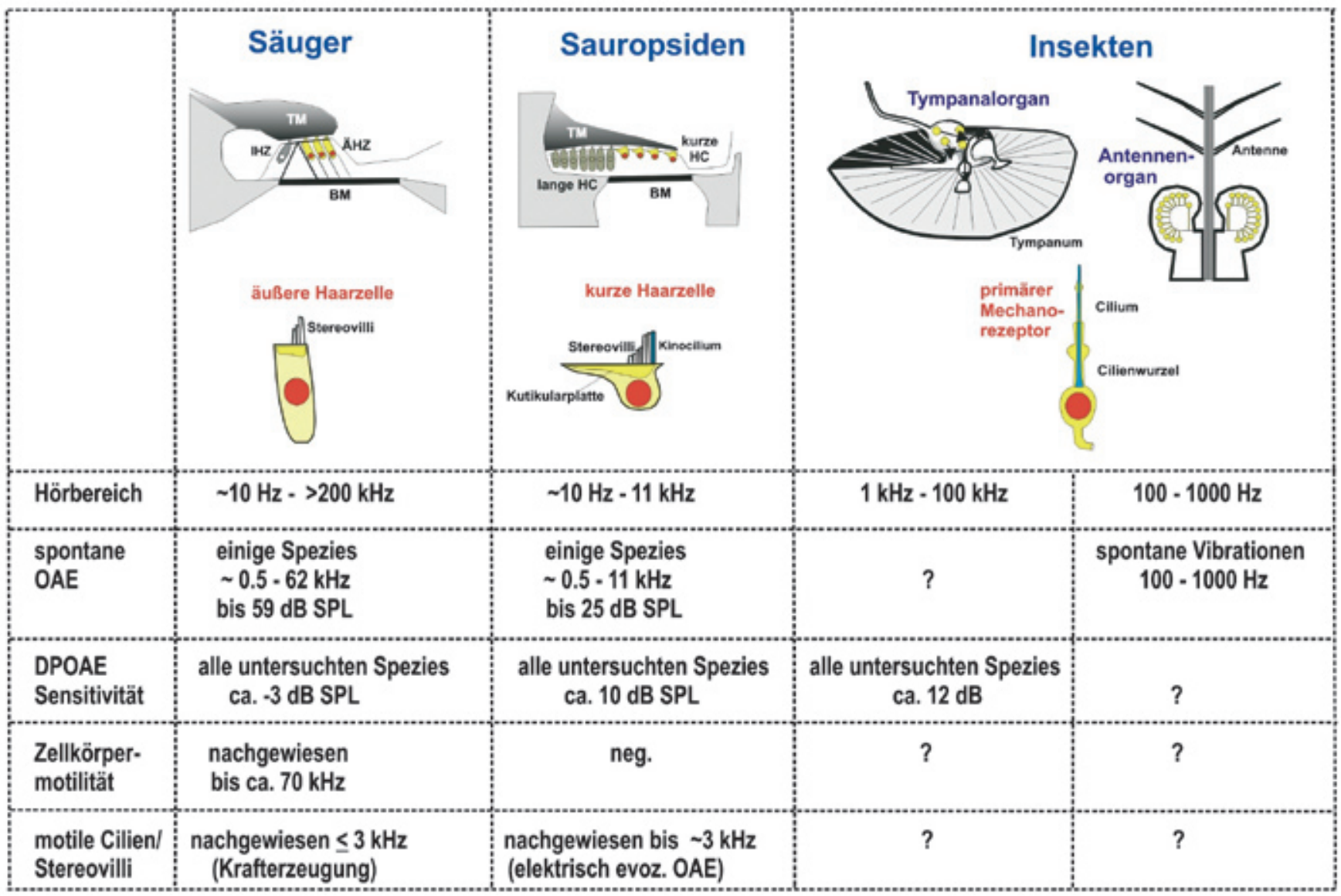

Abb. 5: Wichtige anatomische und bioakustische Parameter im Vergleich von Säuger-, Sauropsiden- und Insektenohren. HZ, Haarzelle; ÄHZ, äußere Haarzelle; IHZ, innere Haarzelle; BM, Basilarmembran; TM, Tectorialmembran. Angabe der Hörbereiche erfolgt ohne Berücksichtigung von Infraschallwahrnehmung. Als Sensitivität für DPOAE wird der für die Tiergruppe minimale Stimuluspegel angegeben, der ausreicht zur Erzeugung einer DPOAE von -10 dB SPL. Daten zum Frequenzverhalten aus Frank et al. 1999 (Zellkörpermotilität von ÄHZ), Ricci et al. 2005 (Säuger: Stereocilienbündel), Manley et al. 2001 (elektrisch evozierte OAE in Reptilien).

in Bezug auf hochfrequente Hörverarbeitung bei Nichtsäugern ist die Schleiereule mit einem Hörbereich, der sich bis zu etwa $11 \mathrm{kHz}$ erstreckt. Bei dieser Eule sind auch spontane OAE bei etwa 9-11 kHz messbar (Exkurs 1). Bei Wirbeltieren gibt es also zwei cochleäre Verstärkungsprozesse, ein Haarbündel-Mechanismus, der tiefe Frequenzen abdeckt, und ein ZellsomaMechanismus, der sowohl tiefe als auch sehr hohe Tonfrequenzen verstärken kann.

Insekten haben im tieffrequenten Bereich bis etwa $1000 \mathrm{~Hz}$ einen mechanischen Ohrverstärker, der in Analogie zu den Verhältnissen bei Nichtsäugern auf einem cilienbasierten Mechanismus begründet zu sein scheint. Dieser ist in den Antennenorganen von Drosophila sehr schön demonstriert. Für Tympanalorgane, die wie das Ohr von Säugern sehr hohe Tonfrequenzen bis über $100 \mathrm{kHz}$ verarbeiten können, ist ein mechanischer Verstärker, der auf den Proteinen des Transduktionsapparates im Cilium beruht, noch nicht nachgewiesen (Abbildung
5). Falls die Krafterzeugung ähnlich wie in den Antennenorganen abläuft, dann wäre dieser Verstärkungsmechanismus deutlich schneller als alle anderen potenziellen aktiven Mechanismen, die im Cilium oder in Stereovilli beschrieben sind. Anders als bei den Antennenorganen konnten bei Tympanalorganen bislang keine spontanen Vibrationen oder OAE demonstriert werden. Sie wären der direkteste Hinweis auf eine aktive Mechanik. Nichtsdestoweniger zeigen die DPOAE-Messungen von Tympanalorganen, dass die Sinneszellen zu einer empfindlichen nichtlinearen mechanischen Verarbeitung leiser Schallstimuli in der Lage sind und dass diese Verarbeitung auf die Schwingungseigenschaften des Tympanums zurückwirkt.

Auch wenn ein potenzieller, aktiver Mechanismus nicht direkt am Transduktionsapparat ansetzt, sondern im Zellsoma lokalisiert ist, wie z.B. Prestin beim Säuger, so hängt er dennoch entscheidend von der Transduktion und dem dadurch entstehenden Rezeptorpotenzial ab. Eine spannungsabhängige mechanische Deformation der Zelle, die dazu beiträgt, das Rezeptorpotenzial zu erhöhen, das den Prozess treibt, stellt ein klassisches verstärkendes Rückkopplungssystem dar. Wenn der Verstärkungsfaktor im Rückkopplungszweig nahe 1 ist, neigt ein solches System auch zu spontanen Oszillationen. Die Erzeugung von DPOAE bei Säugern ist gut modellierbar, wenn man als nichtlineares Element die Kennlinie der Transduktionsströme in Haarsinneszellen verwendet. Derartige Sförmige Transduktionskennlinien werden üblicherweise mit Boltzman-Funktionen dargestellt und reflektieren die pegelabhängigen Eigenschaften von DPOAE gut. Für die realitätsnahe Simulation von DPOAE beim Säuger ist bereits eine einzige Boltzman-Funktion ausreichend (Lukashkin et al. 2002). Vergleicht man nun die Pegelabhängigkeit von DPOAE bei Tympanalorganen mit derjenigen der Säugercochlea und simuliert diese Pegelabhängigkeit mit 

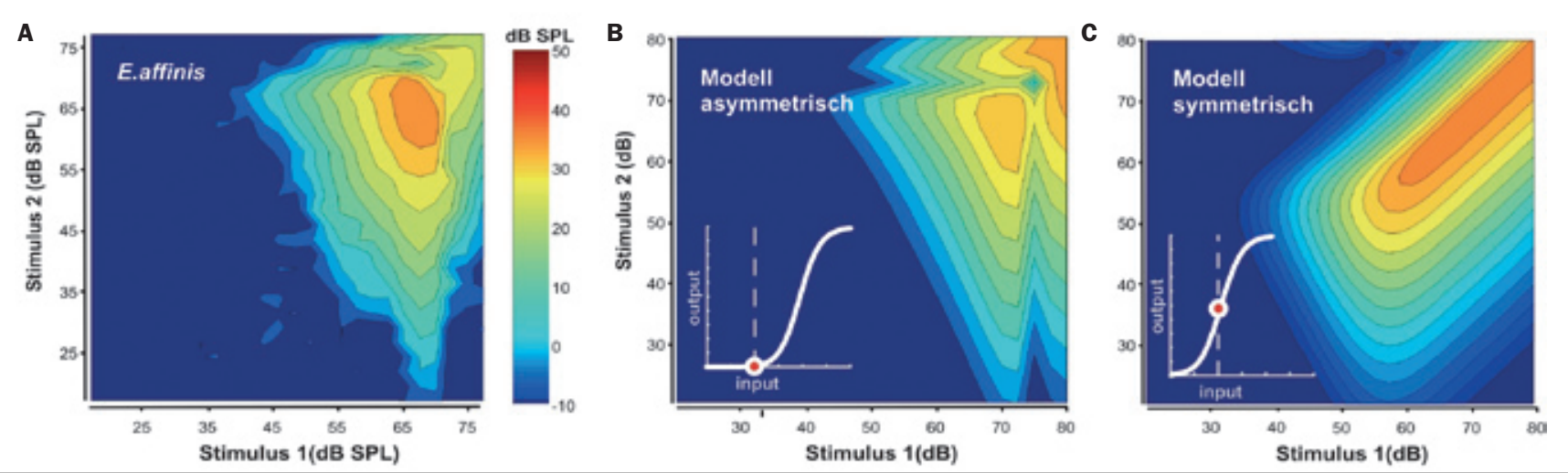

Abb. 6: DPOAE-Erzeugung beim Nachtfalter E. affinis (A) und in einem Modell, das auf einer nichtlinearen Boltzman-Funktion beruht (B, C). Derartige Funktionen werden verwendet, um Transduktionseigenschaften von Wirbeltierhaarzellen zu beschreiben. Dargestellt ist die DPOAE-Amplitude (farbcodiert) in Abhängigkeit von der Amplitude der beiden Reintonstimuli . Die Insektendaten sind gut repräsentiert durch das Modell, wenn der Arbeitspunkt oder Nullpunkt der Boltzmann-Funktion stark asymmetrisch liegt (siehe rote Markierung im Inset B). Die bei symmetrischer Lage des Arbeitspunktes simulierten DPOAE (C) sind ähnlich zu Datensätzen aus dem Säugerohr (verändert nach Kössl und Coro 2006).

einer Boltzman-Funktion, dann stößt man auf einen deutlichen Unterschied zwischen beiden Tiergruppen. Im Falle des Tympanalorgans von Nachtfaltern ergibt sich bei Variation der beiden Stimuluspegel ein dreieckig geformter DPOAE-Anregungsbereich (Abbildung 6A), der durch eine BoltzmannFunktion simulierbar ist (Abbildung 6B), allerdings nur dann, wenn der Nullpunkt oder Arbeitspunkt der Funktion stark asymmetrisch liegt. Verwendet man eine symmetrische Boltzman-Funktion, ist der DPOAE-Anregungsbereich anders geformt (Abbildung 6C) und gleicht demjenigen, der bei Säugern messbar ist (siehe z.B. Kummer et al. 2000). Auch direkt in tympanalen Sinneszellen von Heuschrecken gemessene Rezeptorpotenziale zeigen nur eine Depolarisation, aber keine Hyperpolarisation bei akustischer Reizung (Hill 1983), was ebenfalls durch eine stark asymmetrische Kennlinie erklärbar ist. Das einzelne Cilium von skolopidialen Sinnesneuronen scheint also bei der Transduktion etwas andere Randbedingungen zu erzeugen, als dies in Haarsinneszellen der Fall ist, obwohl im Transduktionsprozess ähnliche Proteine involviert sind.

\section{Literatur}

Göpfert, M.C. (2007): Drosophila-Antenne gewährt Einblicke in grundlegende Mechanismen des Hörens. Neuroforum 4/2007: 122-126.

Kössl, M. und Boyan, G.S. (1998): Acoustic distortion products from the ear of a grasshopper. $J$ Acoust Soc Am 104: 326-335.

Kössl, M., Coro, F., Seyfarth, E.-A. und Nässig, W.A. (2007): Otoacoustic emissions from insect ears having just one auditory neuron. $J$ Comp Physiol A 193: 909-915.
Möckel, D., Seyfarth, E.-A. und Kössl, M. (2007): The generation of DPOAEs in the locust ear is contingent upon the sensory neurons. J Comp Physiol A 193: 871-879.

Windmill, J.F.C., Göpfert, M.C. und Robert, D. (2005): Tympanal travelling waves in migratory locusts. J Exp Biol 208: 157-168.

Eine ausführliche Literaturliste kann bei den Autoren angefordert werden.

\section{Danksagung}

Wir danken Frank Coro für anregende Diskussionen und Kerstin Röhr für die Vorlage zu Abbildung 1A. Unsere Arbeiten werden von der Deutschen Forschungsgemeinschaft unterstützt.

\section{Kurzbiografien}

Manfred Kössl: Studium der Biologie an der LMU München und der Universität Tübingen. Diplom- und Doktorarbeit an der LMU München in der Abteilung von Prof. Gerhard Neuweiler, betreut von Dr. Marianne Vater. DFG-Postdoc an der University of Sussex, Brighton im Labor von Prof. Ian Russell (1988-1990). Wissenschaftlicher Assistent an der LMU München bei Prof. Gerhard Neuweiler (1990-1997). Habilitation in Neurobiologie 1994. Heisenbergstipendiat 1998-2001. Ab 2001 Professor für Zoologie und Leiter der Abteilung Neurobiologie und Biosensorik am Fachbereich Biowissenschaften der J.W. Goethe-Universität Frankfurt.

Doreen Möckel: Studium der Biologie an der J. W. Goethe-Universität Frankfurt. Seit
2005 Promotionsstudentin in der Abteilung Neurobiologie und Biosensorik unter der Betreuung von Prof. Dr. Manfred Kössl und Prof. Dr. E.-A. Seyfarth, gefördert durch ein Stipendium des Evangelischen Studienwerks e.V. Villigst.

Melanie Weber: Studium der Biologie an der J. W. Goethe-Universität Frankfurt mit abschließender Diplomarbeit in der Abteilung Neurobiologie und Biosensorik. Seit 2005 Promotionsstudentin unter der Betreuung von Prof. Dr. E.-A. Seyfarth und Prof. Dr. Manfred Kössl, gefördert durch ein Stipendium der Jürgen-Manchot-Stiftung.

Ernst-August Seyfarth: Biologie- und Psychologiestudium in Marburg, Massachusetts und München (LMU). Diplom und Promotion bei Friedrich G. Barth an der LMU; ab 1975 wissenschaftlicher Mitarbeiter am Fachbereich Biowissenschaften der J.W. Goethe-Universität Frankfurt; seit 1998 apl. Professor eben dort. Arbeiten zur Mechanorezeption und Neuroethologie von Arthropoden (speziell Spinnen) sowie zur biografischen Geschichte der Neurobiologie. Mehrere Forschungsaufenthalte in Mexiko, Kanada, Australien und USA.

\section{Korrespondenzadresse}

\section{Prof. Dr. Manfred Kössl}

Institut für Zellbiologie und

Neurowissenschaft

J.W. Goethe-Universität

Siesmayerstr.70A

60323 Frankfurt/Main

Tel.: $\quad+49$ (0) 6979824761

E-Mail:koessl@bio.uni-frankfurt.de 